\title{
Solution Multiplicity of Smoke Flows in a Simple Building
}

\author{
JIAN GONG and YUGUO LI \\ Department of Mechanical Engineering \\ The University of Hong Kong, Pokfulam, Hong Kong SAR, China
}

\section{ABSTRACT}

For a simple single-zone building on fire with two openings and opposing wind, a theoretical analysis of smoke flow rate and smoke flow direction is carried out. With the same setting of building geometry and physical conditions at steady state, three solutions of smoke flows are identified. Fundamental concepts of nonlinear dynamics are used to show that two of these solutions are stable. One stable solution allows the establishment of the two-layer flow pattern with an upward smoke flow, which may be considered relatively safe in terms of control of smoke flows. The other stable solution is a potentially unsafe situation of downward smoke flow pattern with smoke filling the entire building. We believe that existence of solution multiplicity of smoke flows in this simple building demonstrates that a further study of more complex and realistic situations is necessary.

KEYWORDS: smoke, smoke flows, solution multiplicity, opposing winds, buoyancy flows

\section{NOMENCLATURE LISTING}

A area of the opening $\left(\mathrm{m}^{2}\right)$

$A^{*} \quad$ effective opening area $\left(\mathrm{m}^{2}\right)$

$A_{c} \quad$ floor area of the building $\left(\mathrm{m}^{2}\right)$

$C_{d} \quad$ discharge coefficient

$c_{p} \quad$ specific heat capacity $(\mathrm{J} / \mathrm{kg} \mathrm{K})$

E a constant $(=0.196)$

Fr Froude number

$g$ acceleration of gravity $\left(\mathrm{m} / \mathrm{s}^{2}\right)$

$H$ height of the building (m)

$H^{*} \quad$ non-dimensional height of smoke

discontinuity plane

$h_{d} \quad$ discontinuity plane height (m)

$k \quad$ ratio of effective top opening over

$k \quad$ effective bottom opening

$M \quad$ mass of indoor air of building (kg)

$m$ mass flow rate $(\mathrm{kg} / \mathrm{s})$

$m_{A} \quad$ mass flow rate involving the effect

$m_{A} \quad$ of opening area of building $(\mathrm{kg} / \mathrm{s})$ mass flow rate involving the effect

$m_{H} \quad$ of height of building $(\mathrm{kg} / \mathrm{s})$

$m_{p} \quad$ mass flow rate of plume $(\mathrm{kg} / \mathrm{s})$

$m_{Q} \quad$ mass flow rate induced by convective heat release rate $(\mathrm{kg} / \mathrm{s})$

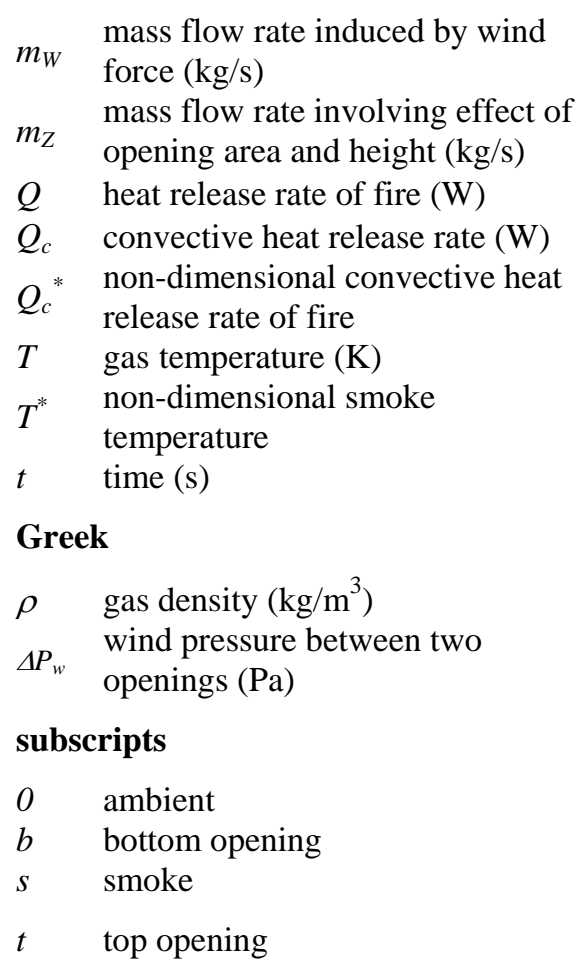

\section{INTRODUCTION}

Smoke exhibits a severe risk to people in a building fire [1, 2]. There have been a number of significant studies to understand and predict smoke flows in building fires. In addition to experimental investigation, the other methods of modeling smoke flows are mathematical techniques including the zone model and the field model. The zone model typically divides a room into two layers, that is, an upper hot smoke layer and a lower relatively cool gas layer. Thomas et al. [3] employed a two-layer model to analyze smoke flows produced by a small fire in horizontal and pitched roof venting, and compared these results with experimental data. Thereafter, some other investigators also used the two-layer model to predict smoke flows in buildings [4-7]. For example, Jones and co-workers [5] developed the most widely-used zone- 
based model, CFAST (consolidated fire and smoke transport). The field model is also known as Computational Fluid Dynamics (CFD). Markatos et al. [8] took advantage of finite difference method to simulate smoke flows in an enclosure. McGrattan et al. [9] considered smoke flows in an enclosure using a large eddy simulation approach. This algorithm was the primary theory in the so-called Fire Dynamics Simulator (FDS), which is a widely-used free software of the field-based smoke flow model.

The solutions of smoke flows can be used as a predictive and guidable tool in smoke management systems. Among the solutions, smoke flow direction, smoke flow rate and smoke temperature are the most important parameters in smoke flow design. Comparatively speaking, the smoke flow direction is especially significant and important in smoke spread and human evacuation. The undesirable smoke spread direction may be avoided or changed if the mechanisms of smoke flow are well understood and controlled.

The multiple smoke flow directions represent multiple smoke flow solutions. On the one hand, a number of numerical studied by Nitta [10-12] suggested that multiple smoke flow solutions could exist in simple buildings under same settings of heat source and wind force. A Newton-Raphson method was used for ventilation network model to analyze smoke direction in a building on fire. However, these studies only assumed that the smoke in each room was fully mixed and the thermal stratification was ignored. Also, the stability of multiple solutions of smoke flows was not analyzed. On the other hand, existence of multiple solutions is widely known in the closely related field of natural ventilation in buildings. Maeda and Matsuura [13] were among the first scholars to experimentally identify that there existed more than one natural ventilation mode in a building. Li and Delsante [14] considered a single-zone building with two openings under the combined action of thermal force and wind force. Their analysis showed there were three solutions of airflow direction in the building and two were stable. Li and others [15] used a smallscale experiment to verify the multiple solutions. Meanwhile, Hunt and Linden [16] gave almost identical evidence for the solution multiplicity in natural ventilation.

The driving forces of smoke flows in buildings are the pressure differences due to the presence of fire, stack pressure, wind force and mechanical system [17]. It would be expected that the multiple solutions would exist in smoke flows as that in natural ventilation. However, it seems that researchers in fire community have not paid much attention to the solution multiplicity of smoke flows to our best knowledge. In this paper, we employ a zone model to analyze smoke flows in a single-zone building with two openings under the condition of an opposing wind. The mass flow rate is used to take into account of large density variation of smoke under thermal stratification. We apply the basic concepts of nonlinear dynamics to evaluate the stability of the multiple solutions.

\section{A SIMPLE BUILDING MODEL}

Consider a simple single-zone building with a top opening at one side and a bottom opening at the opposite side, as shown in Fig. 1. The openings are relatively small in opening height. There is a fire on the floor and the smoke flow is upwards. Here there are an upper smoke layer and a lower gas layer. When we consider the effect of an opposing wind, two possible situations are expected, namely when buoyancy force exceeding the opposing wind force (weak wind) with an upward smoke flow, and the opposing wind force exceeding the buoyancy force (strong wind) with a downward smoke flow, as shown in Fig. 2.

To simplify the problem, the following assumptions are made:

(1) The fire or smoke is considered as a physical not a chemical phenomenon, so we just take heat into account in our analysis.

(2) All walls are perfectly adiabatic and we ignore the effect of thermal radiation.

(3) The heat release rate of fire $Q$ does not change with time, so does the convective heat release rate $Q_{c}$.

(4) The flows through both openings are unidirectional and the simple Bernoulli's equation is used.

(5) The pressure variation in the building is small compared to the ambient pressure, so the air pressure can be approximately considered as a constant. Thus, the ideal gas equation can be written as $\rho_{0} T_{0}=\rho_{s} T_{s}$.

(6) Ambient air and smoke are both considered as ideal gases taking the same specific heat capacity at constant pressure $c_{p}$, which does not change with temperature. 
(7) The top opening is located at the height $H$ and the bottom opening is located at height zero. The floor area of the building $A_{c}$ is constant with height.

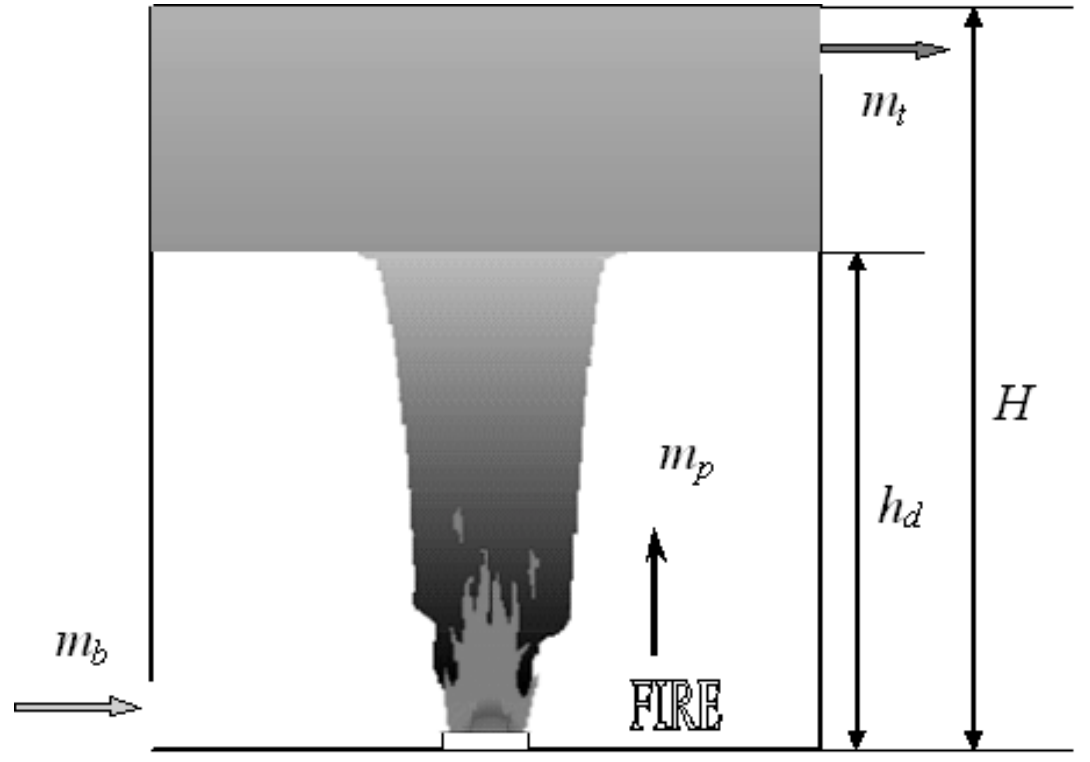

Fig. 1. Illustration of a single-zone building with a bottom fire

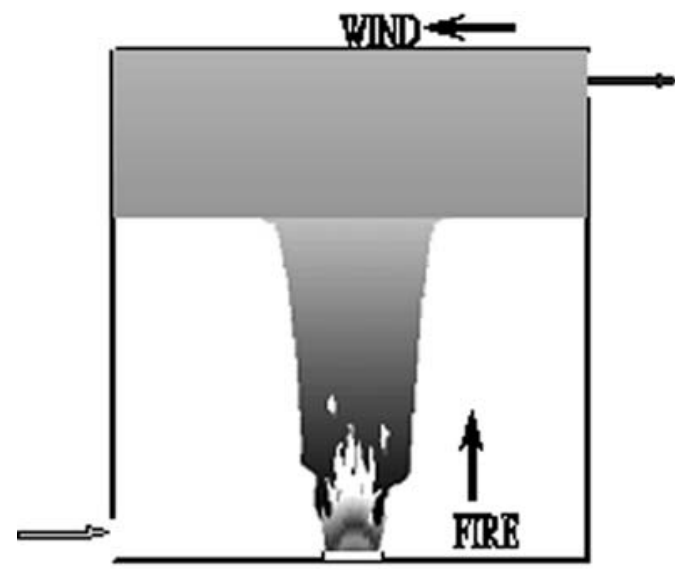

(A)

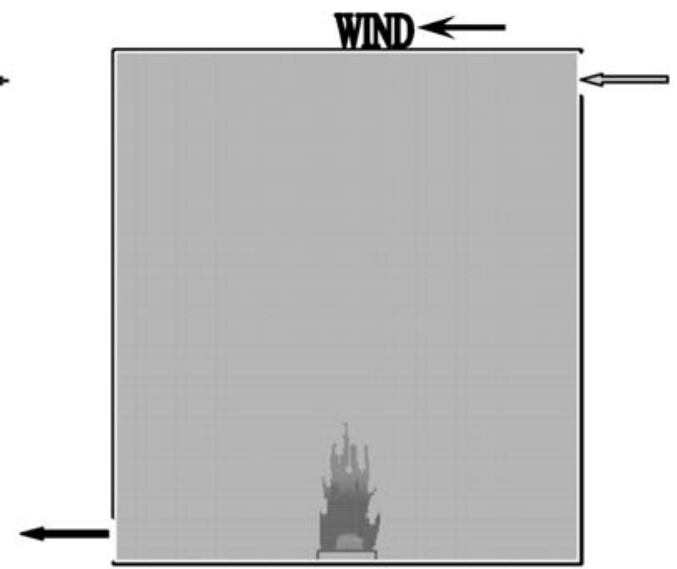

(B)

Fig. 2. Two situations with an opposing wind; (A) a weak wind with an upward smoke flow and two layers; (B) a strong wind with a downward smoke flow and the smoke is fully mixed in the building.

Where there is a weak opposing wind, thermal buoyancy dominates and the direction of smoke flows is upwards. We adopt zone model and the temperature of lower air layer equals the ambient temperature $T_{0}$. The mass conservation and energy conservation equations of the upper layer are as follows:

$\frac{d\left[\rho_{s} A_{c}\left(H-h_{d}\right)\right]}{d t}=m_{p}-m_{t}$

$c_{p} \frac{d\left[\rho_{s} A_{c}\left(H-h_{d}\right) T_{s}\right]}{d t}=Q_{c}+c_{p} m_{p} T_{0}-c_{p} m_{t} T_{s}$

The mass conservation equation of the lower layer is 
$\frac{d \rho_{0} A_{c} h_{d}}{d t}=m_{b}-m_{p}$

Using the simple Bernoulli’s equation and the pressure loop equation, we have

$\left(\rho_{0}-\rho_{s}\right) g\left(H-h_{d}\right)-\Delta P_{w}=\frac{m_{b}^{2}}{\left(C_{d} A_{b}\right)^{2} 2 \rho_{0}}+\frac{m_{t}^{2}}{\left(C_{d} A_{t}\right)^{2} 2 \rho_{s}}$

Here $\Delta P_{w}$ is the wind pressure difference between the two openings, which is always thought as nonnegative. Then we use the mass flow rate equation of plume proposed by Heskestad [18], which is suitable for strong plumes and we only consider the first term for simplicity.

$m_{p}=E\left(\frac{g \rho_{0}^{2}}{c_{p} T_{0}}\right)^{1 / 3} Q_{c}^{1 / 3} h_{d}^{5 / 3}$

Here $E=0.196$ [18]. After some manipulation, the governing equations are simplified as follows:

$$
\begin{aligned}
& c_{p} \rho_{0} T_{0} A_{c}\left(H-h_{d}\right) \frac{d T_{s}}{d t}=T_{s}\left[Q_{c}+c_{p} E\left(\frac{g \rho_{0}^{2}}{c_{p} T_{0}}\right)^{1 / 3} Q_{c}^{1 / 3} h_{d}^{5 / 3}\left(T_{0}-T_{s}\right)\right] \\
& A_{c} \rho_{0} \frac{d h_{d}}{d t}=m_{b}-E\left(\frac{g \rho_{0}^{2}}{c_{p} T_{0}}\right)^{1 / 3} Q_{c}^{1 / 3} h_{d}^{5 / 3} \\
& Q_{c}+c_{p} m_{b} T_{0}-c_{p} m_{t} T_{s}=0 \\
& \rho_{0} \frac{T_{s}-T_{0}}{T_{s}} g\left(H-h_{d}\right)-\Delta P_{w}=\frac{m_{b}^{2}}{\left(C_{d} A_{b}\right)^{2} 2 \rho_{0}}+\frac{T_{s} m_{t}^{2}}{\left(C_{d} A_{t}\right)^{2} 2 \rho_{0} T_{0}}
\end{aligned}
$$

When there is a strong opposing wind, the dominating wind induces a strong mixing in the building. The direction of smoke flows is downwards. We assume that the indoor smoke is fully mixed. Based on mass and energy conservation, and the simple Bernoulli's equation and the pressure loop equation, we have

$$
\begin{aligned}
& \rho_{0} A_{c} H T_{0} \frac{d T_{s}}{d t}=T_{s}^{2}\left(m_{b}-m_{t}\right) \\
& Q_{c}+c_{p} m_{t} T_{0}-c_{p} m_{b} T_{s}=0 \\
& \Delta P_{w}-\rho_{0} \frac{T_{s}-T_{0}}{T_{s}} g H=\frac{m_{b}^{2} T_{s}}{\left(C_{d} A_{b}\right)^{2} 2 \rho_{0} T_{0}}+\frac{m_{t}^{2}}{\left(C_{d} A_{t}\right)^{2} 2 \rho_{0}}
\end{aligned}
$$




\section{STEADY-STATE ANALYSIS}

\section{Steady-state Solutions}

In this section, the solutions (steady states) of the governing equations are analyzed. The steady-state solutions correspond to the fixed points of a dynamical system, which means that it does not change with time. We can obtain the steady-state solutions by letting the derivative terms versus time equal zero. A stable fixed point can damp out all sufficiently small perturbation with time, while perturbation grows with time at an unstable fixed point [19]. Here we define some group parameters to simplify the steady-state equations:

$$
m_{Q}=\frac{Q_{c}}{c_{p} T_{0}}
$$

$m_{W}=\left(C_{d} A^{*}\right) \sqrt{2 \rho_{0} \Delta P_{w}}$

$m_{Z}=\left(C_{d} A^{*}\right) \sqrt{2 \rho_{0}^{2} g H}$

$m_{A}=\left[2\left(C_{d} A^{*}\right)^{2} \rho_{0}^{8 / 5} g^{4 / 5} \frac{1}{E^{3 / 5}}\right]^{5 / 8}$

$m_{H}=\left(E \rho_{0}^{2 / 3} g^{1 / 3} H^{5 / 3}\right)^{3 / 2}$

$m_{H}^{2 / 5} m_{A}^{8 / 5}=m_{Z}^{2}$

$k=\frac{C_{d} A_{t}}{C_{d} A_{b}}$

Where $C_{d} A^{*}=\frac{\left(C_{d} A_{b}\right)\left(C_{d} A_{t}\right)}{\sqrt{\left(C_{d} A_{b}\right)^{2}+\left(C_{d} A_{t}\right)^{2}}}$.

In defining the parameters $m_{Q}, m_{W}, m_{Z}, m_{A}, m_{H}$, we intentionally ensure that they all have a unit of mass flow rate $(\mathrm{kg} / \mathrm{s})$ with some physical meanings. $m_{Q}$ quantifies mass flow rate induced by convective heat release rate of fire, $m_{W}$ measures mass flow rate driven by wind force, $m_{A}$ and $m_{H}$ mean mass flow rate involving the effect of opening area and height of building, and $m_{Z}$ means mass flow rate involving the effect of a fixed building. There is a relationship amongst $m_{A}, m_{H}$ and $m_{Z}$ as defined in Eq. 18. $k$ is the ratio of effective top opening area to effective bottom opening area and we assume $k=1$ for convenience in the following analysis.

Consequently, on the one hand at the steady state with a weak wind $\left(d T_{s} / d t=d h_{d} / d t\right)$, we can obtain the following equation of the mass flow rate of smoke.

$m^{3}+\frac{3}{2} m_{Q} m^{2}+\frac{1}{2} m_{Q}^{2} m+m_{A}^{8 / 5} m_{Q}^{4 / 5} m^{3 / 5}-m_{Z}^{2} m_{Q}+m_{W}^{2}\left(m_{Q}+m\right)=0$ 
Here $m=m_{b}=m_{t}=m_{p}$. The solutions can be presented in a non-dimensional graph. For example, the equation of the mass flow rate ratio $\left(y=m / m_{W}\right)$ versus heat source $\left(x=m_{Q} / m_{W}\right)$ is

$$
y^{3}+\frac{3}{2} x y^{2}+\left[\frac{1}{2} x^{2}+1\right] y+\left(\frac{m_{A}}{m_{W}}\right)^{8 / 5} x^{4 / 5} y^{3 / 5}-\left[\left(\frac{m_{Z}}{m_{W}}\right)^{2}-1\right] x=0
$$

The $x$ axis is

$$
\frac{m_{Q}}{m_{W}}=\frac{1}{F r}\left(Q_{c}^{*}\right)^{2 / 3}\left(\frac{H^{2}}{C_{d} A^{*}}\right)^{1 / 3}
$$

Here $\mathrm{Fr}$ is the Froude number defined in [20], $Q_{c}{ }^{*}$ is the non-dimensional convective heat release rate defined in [2], and $C_{d} A^{*} / H^{2}$ is the non-dimensional opening area.

$$
\begin{gathered}
F r=\frac{\sqrt{2 \Delta P_{w} / \rho_{0}}}{\left(\frac{Q_{c} g}{c_{p} \rho_{0} T_{0} H}\right)^{1 / 3}} \\
Q_{c}^{*}=\frac{Q_{c}}{c_{p} \rho_{0} T_{0}\left(C_{d} A^{*}\right) \sqrt{g H}}
\end{gathered}
$$

We choose some typical values $m_{A} / m_{W}=1.1, m_{Z} / m_{W}=1.25,1.5$ to reveal the relationship and the mass flow rate ratio curves are shown in Fig. 3.

Similarly, the equation of the mass flow rate ratio $\left(y=m / m_{Q}\right)$ versus wind force $\left(x=m_{W} / m_{Q}\right)$ is

$$
y^{3}+\frac{3}{2} y^{2}+\left(\frac{1}{2}+x^{2}\right) y+\left(\frac{m_{A}}{m_{Q}}\right)^{8 / 5} y^{3 / 5}-\left(\frac{m_{Z}}{m_{Q}}\right)^{2}+x^{2}=0
$$

We choose some typical values $m_{A} / m_{Q}=1.5, m_{Z} / m_{Q}=4,6$ to reveal the relationship, and the mass flow rate ratio curves are shown in Fig. 4.

On the other hand, at the steady state with a strong wind $\left(d T_{s} / d t=0\right)$, we can obtain the following equation of the mass flow rate of smoke.

$$
m^{3}+\frac{3}{2} m_{Q} m^{2}+\frac{1}{2} m_{Q}^{2} m+m_{Z}^{2} m_{Q}-m_{W}^{2}\left(m+m_{Q}\right)=0
$$

Here $m=m_{b}=m_{t}$. Then, the equation of the mass flow rate ratio $\left(y=m / m_{W}\right)$ versus heat source $\left(x=m_{Q} / m_{W}\right)$ is

$$
y^{3}+\frac{3}{2} x y^{2}+\left(\frac{1}{2} x^{2}-1\right) y+\left(\frac{m_{Z}}{m_{W}}\right)^{2} x-x=0
$$

We also choose the same values $m_{Z} / m_{W}=1.25,1.5$ and the mass flow rate ratio curves are shown in Fig. 3. Similarly, the equation of the mass flow rate ratio $\left(y=m / m_{Q}\right)$ versus wind force $\left(x=m_{W} / m_{Q}\right)$ is 
$y^{3}+\frac{3}{2} y^{2}+\left(\frac{1}{2}-x^{2}\right) y+\left(\frac{m_{Z}}{m_{Q}}\right)^{2}-x^{2}=0$

We also choose the same values $m_{Z} / m_{Q}=4,6$ and the mass flow rate ratio curves are shown in Fig. 4.

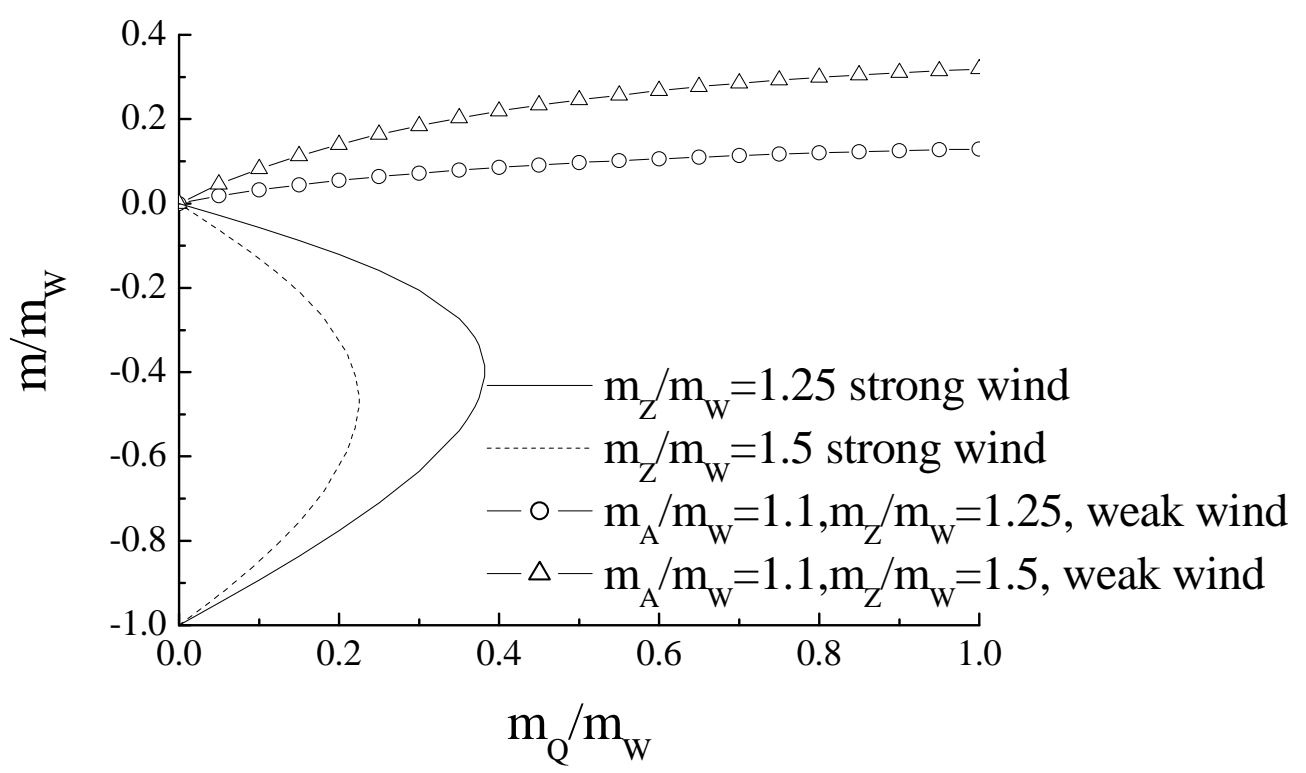

Fig. 3. Curves of mass flow rate ratio of smoke versus heat source for given values of $m_{A} / m_{W}$ and $m_{Z} / m_{W}$

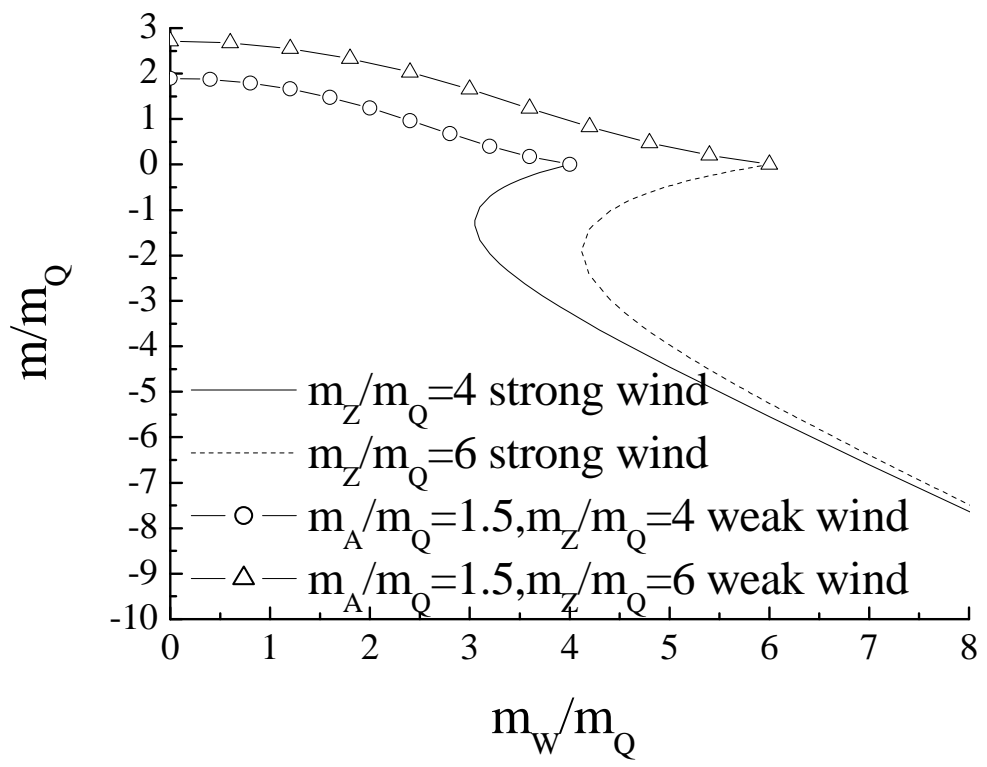

Fig. 4. Curves of mass flow rate ratio of smoke versus wind force for given values of $m_{A} / m_{Q}$ and $m_{Z} / m_{Q}$ 
In Figs. 3-4 we adopt an upward smoke flow as positive and a downward smoke flow as negative. In Fig. 3, there are three solutions for a range of $m_{Q} / m_{W}$ values, and two of them corresponding to a strong wind and one corresponding to a weak wind. For example, values from zero to near 0.4 in curve of solid line and in curve of solid line with circle symbol. It should be noted that $m / m_{W}=0$ corresponds to $m_{Q} / m_{W}=0$ based on the governing equations. However, $m_{Q} / m_{W}=0$ also means the absence of fire, so the "smoke" flow rate equals the downward air flow rate induced by wind alone, namely $m / m_{W}=-1$. Thus, we cannot obtain $\mathrm{m} / \mathrm{m}_{Q}=0$ with a weak wind.

In Fig. 4, there are three solutions for a range of $m_{W} / m_{Q}$ values, and two of them corresponding to a strong wind and one corresponding to a weak wind. The following extreme case is noted. Let us take example of $m_{A} / m_{Q}=1.5, m_{Z} / m_{Q}=4$ with a weak wind. When $m / m_{Q}=0$, it corresponds to $m_{W} / m_{Q}=4$ according to the governing equations. However, $m_{W} / m_{Q}=4$ and $m_{Z} / m_{Q}=4$ means $m_{W}=m_{Z}$, which expresses the opposing wind is strong enough to cause a downward flow regardless of fire, namely $m / m_{Q}<0$ according to our definition. Thus, we cannot obtain $m / m_{Q}=0$ with a weak wind.

\section{Stability of Solutions}

Now, we analyze the stability of these three steady-state solutions.

For the situation of a weak opposing wind, the governing Eqs. 6-9 can be generalized into two nondimensional ordinary differential equations $(k=1)$ :

$$
\begin{aligned}
\frac{M}{m_{W}} \frac{d H^{*}}{d t}= & \frac{T^{* 1 / 2}}{1+T^{*}} \sqrt{2 \frac{T^{* 2}-1}{T^{*}}\left(1-H^{*}\right)\left(\frac{m_{Z}}{m_{W}}\right)^{2}-2\left(1+T^{*}\right)-\left(\frac{m_{Q}}{m_{W}}\right)^{2}} \\
& -\frac{m_{Q} / m_{W}}{1+T^{*}}-\left(\frac{m_{H}}{m_{W}}\right)^{2 / 3}\left(\frac{m_{Q}}{m_{W}}\right)^{1 / 3} H^{* 5 / 3} \\
\frac{M}{m_{W}} \frac{d T^{*}}{d t}= & \frac{T^{*}}{1-H^{*}}\left[\frac{m_{Q}}{m_{W}}+\left(\frac{m_{H}}{m_{W}}\right)^{2 / 3}\left(\frac{m_{Q}}{m_{W}}\right)^{1 / 3}\left(1-T^{*}\right) H^{* 5 / 3}\right]
\end{aligned}
$$

Where $M=\rho_{0} A_{c} H, T^{*}=T_{s} / T_{0}, H^{*}=h_{d} / H$. Besides, the initial condition should satisfy

$$
2\left(\frac{m_{\mathrm{Z}}}{m_{W}}\right)^{2}\left(1-H^{*}\right)\left(T^{*}-1\right)-2 T^{*}>\left(\frac{m_{Q}}{m_{W}}\right)^{2}
$$

The physical meaning of this inequality represents that ambient air flow through bottom opening should be rightward, which corresponds to a relatively small convective heat release rate of fire. If it is relatively large convective heat release rate, it is a transit situation,which will be analyzed in the near future. The stability of the fixed points can be determined by the signs of real part of eigenvalues in the linearization matrix. If all real parts of the eigenvalues are positive, it is an unstable fixed point. If all real parts are negative, it is a stable fixed point. However, if some signs are positive while the others are negative, the fixed point is a saddle, and if one of the eigenvalues has zero real part, there is a bifurcation in the point [21]. Thus, we can judge the stability by the linearization matrix:

$$
G=\left(\begin{array}{ll}
\frac{\partial f_{1}}{\partial H^{*}} & \frac{\partial f_{1}}{\partial T^{*}} \\
\frac{\partial f_{2}}{\partial H^{*}} & \frac{\partial f_{2}}{\partial T^{*}}
\end{array}\right)_{\left(H_{0}^{*}, T_{0}^{*}\right)}
$$


Here $f_{1}=\left(M / m_{W}\right)\left(d H^{*} / d t\right), f_{2}=\left(M / m_{W}\right)\left(d T^{*} / d t\right), G$ is called the Jacobian matrix at the fixed point $\left(H_{0}{ }^{*}, T_{0}{ }^{*}\right)$. Let $m_{Z} / m_{W}=1.25, m_{A} / m_{W}=1.1, m_{Q} / m_{W}=0.3$, then the numerical fixed point is $(0.1949,5.1927)$ and the numerical eigenvalues of matrix $G$ at this point are -8.7992 and -1.1484 , so the solution is stable.
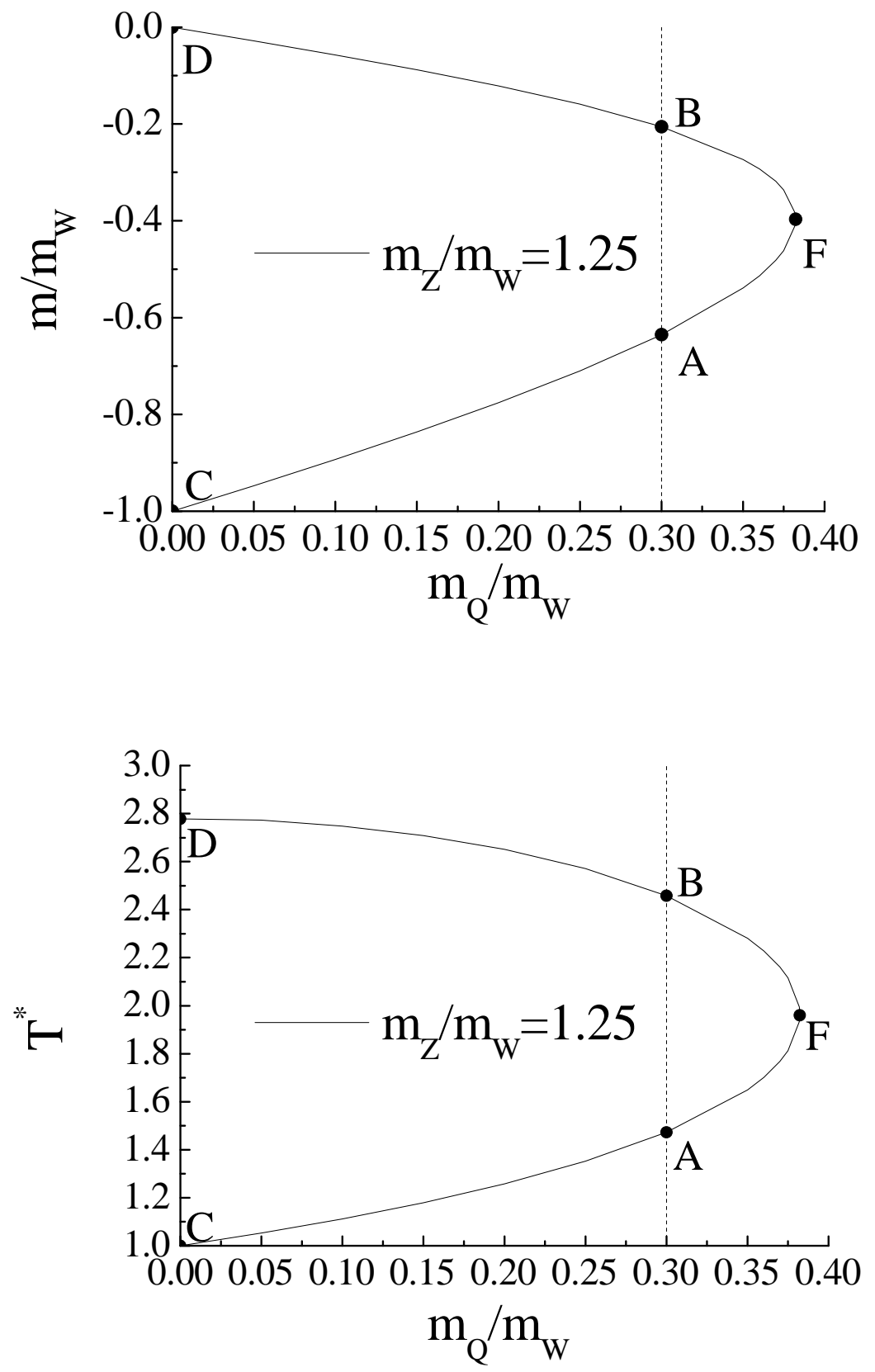

Fig. 5. Curves of mass flow rate and temperature ratio versus heat source with strong wind for $m_{\mathbb{Z}} / m_{W}=1.25$

For the situation of a strong opposing wind, the governing Eqs. 10-12 can be derived into one nondimensional ordinary differential equation $(k=1)$ : 


$$
\frac{M}{m_{W}} \frac{d T^{*}}{d t}=\frac{T^{* 3 / 2}\left(1-T^{*}\right)}{1+T^{*}} \sqrt{2\left(1+T^{*}\right)-2 \frac{T^{* 2}-1}{T^{*}}\left(\frac{m_{Z}}{m_{W}}\right)^{2}-\left(\frac{m_{Q}}{m_{W}}\right)^{2}}+\frac{2 T^{* 2}}{1+T^{*}} \frac{m_{Q}}{m_{W}}
$$

Besides, the initial condition should satisfy

$$
2 T^{*}-2\left(\frac{m_{Z}}{m_{W}}\right)^{2}\left(T^{*}-1\right)>\left(\frac{m_{Q}}{m_{W}}\right)^{2}
$$

This inequality ensures $m_{t}>0$. When $m_{Z} / m_{W}=1.25$ and $m_{Q} / m_{W}=0.3$, there are two solutions of mass flow rate ratio $m / m_{W}$ and temperature ratio $T^{*}=T_{s} / T_{0}$, namely the fixed points $A$ and $B$ as shown in Fig. 5.

For a system consisting of only one ordinary differential equation, we can evaluate the stability according to slope at the fixed points as shown in Fig. 6. If the slope is positive, it is an unstable fixed point. While the slope is negative, it is a stable fixed point [19]. It should be noted that the fixed points $A$ and $B$ in Figs. 5-6 are mutually corresponding. On the basis of Fig. 6, the fixed point $A$ is stable due to the negative slope and the fixed point $B$ is unstable due to the positive slope. Namely, the solution $A$ is stable while the solution $B$ is unstable. Therefore, the branch $C F$ is stable while the branch $D F$ is unstable, as shown in Fig. 5. The value of critical point $F$ can be obtained by using fundamental mathematical calculation, but the form is complex in analytical form. Here the numerical $x$-axis value $m_{Q} / m_{W}$ of point $F$ is 0.3823 .

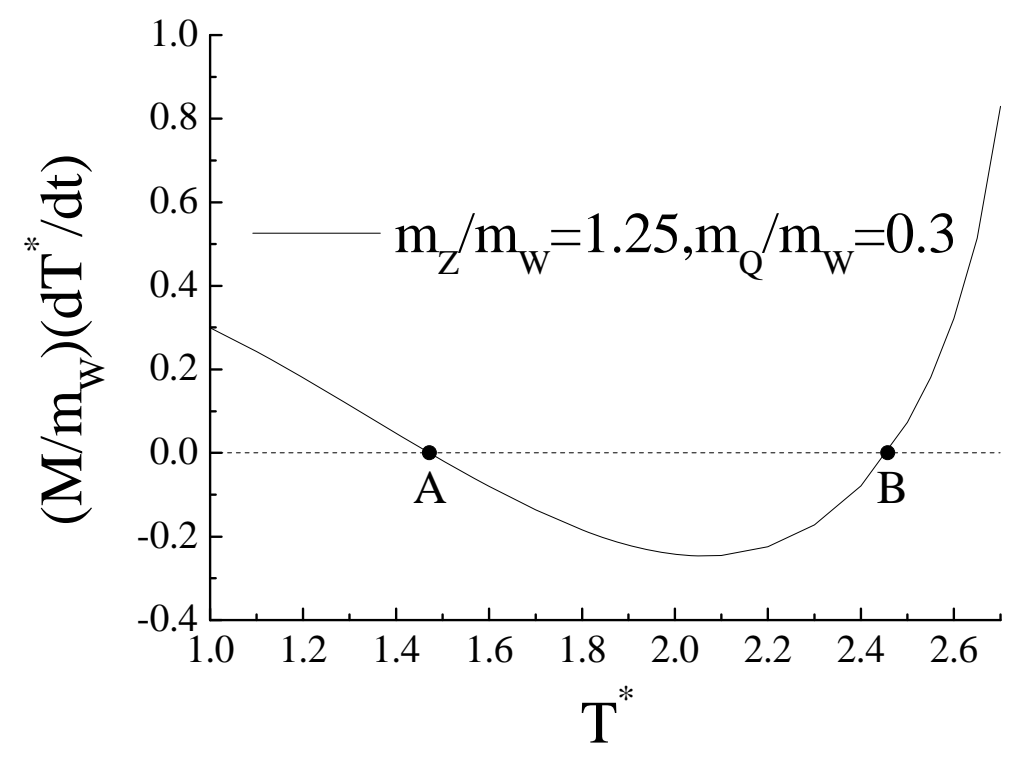

Fig. 6. Curve of $\left(M / m_{W}\right)\left(d T^{*} / d t\right)$ versus temperature ratio for $m_{Z} / m_{W}=1.25$ and $m_{Q} / m_{W}=0.3$

\section{CONCLUSIONS}

We show that there are three possible smoke flows for a simple building under the same setting of a fire source and an opposing wind at the steady state, and two of the solutions are exhibited to be stable while the third one is unstable by simple nonlinear dynamical analysis. One of the stable solutions is characterized by an upward smoke flow pattern and a lower ambient air layer, while the second stable solution features a downward smoke flow pattern with indoor smoke being fully mixed. In a building fire, the lower ambient air layer may protect occupants from inhaling harmful smoke if it is sufficiently high. The occupants may escape through the bottom opening, besides, the top opening, which is also known as a static smoke exhaust system, can remove effectively smoke from the building with the upward smoke flow, 
avoiding smoke accumulation in the upper part of the building. Therefore, the first stable solution may be relatively safe for occupants in smoke control and smoke management system, while the second stable solution may contain risk, representing probably a relatively unsafe situation. The existence of solution multiplicity of smoke flows is important and significant in smoke control and smoke management, and the work here may indicate that smoke control is more difficult than we previously expected. Further dynamical analysis and CFD simulations are needed. In particular, we plan to investigate solution multiplicity of smoke flows using hot gas modeling and a small-scale experimental rig.

\section{REFERENCES}

[1] Klote, J.H. and Milke, J.A., Design of Smoke Management Systems, Atlanta: American Society of Heating, Refrigerating and Air-Conditioning Engineers, Inc, 1992.

[2] Drysdale, D., An Introduction to Fire Dynamics ( $\left.2^{\text {nd }} e d\right)$, John Wiley and Sons, Chichester, 1999.

[3] Thomas, P.H., Hinkley, P.L., Theobald, C.R., Simms, D.L., "Investigations into the Flow of Hot Gases in Roof Venting,” Fire Research Technical Paper No.7, Fire Research Station, UK, 1963.

[4] Zukoski, E.E. and Kubota, T., (1980) Two-layer Modeling of Smoke Movement in Building Fires, Fire and Materials 4(1): 17-27, http://dx.doi.org/10.1002/fam.810040103

[5] Jones, W.W. and Forney, G.P., (1993) Improvement in Predicting Smoke Movement in Compartmented Structures, Fire Safety Journal 21:269-297, http://dx.doi.org/10.1016/03797112(93)90017-K

[6] Fu, Z. and Hadjisophocleous, G., (2000) A Two-zone Fire Growth and Smoke Movement Model for Multi-compartment Buildings, Fire Safety Journal 34:257-285, http://dx.doi.org/10.1016/S0379-7112(99)00045-4

[7] Chow, W.K., Li, J., (2001) Simulation on Natural Smoke Filling in Atrium with a Balcony Spill Plume, Journal of Fire Sciences 19:258-283, http://dx.doi.org/10.1106/65LF-H4GA-9EW6-QF8E

[8] Markatos, N.C., Malin, M.R. and Cox, G., (1982) Mathematical Modelling of Buoyancy-induced Smoke Flow in Enclosures, International Journal of Heat and Mass Transfer 25:63-75, http://dx.doi.org/10.1016/0017-9310(82)90235-6

[9] McGrattan, K.B., Baum, H.R. and Rehm, R.G., (1998) Large Eddy Simulations of Smoke Movement, Fire Safety Journal 30:161-178, http://dx.doi.org/10.1016/S0379-7112(97)00041-6

[10] Nitta, K., “Analytical Study on a Variety of Forms of Multi-room Ventilation,” In Proceedings of the International Symposium on Building and Urban Environment Engineering'97, Tianjin, China, 1997, pp. 67-78.

[11] Nitta, K., "Variety Modes and Chaos in Smoke Ventilation by Ceiling Chamber System," In Proceedings of the Sixth International IBPSA Conference, Kyoto, Japan, 1999, pp. 473-480.

[12] Nitta, K., "Variety Modes and Chaos in Natural Ventilation or Smoke Venting System," In Proceedings of the Seventh International IBPSA Conference, Rio de Janeiro, Brazil, 2001, pp. 635-642.

[13] Maeda, T., Matsuura, T., "Experiments on Ventilator,” Research Report of Architecture Planning and Environmental Engineering (Transactions of AIJ), 1952, 19:1-4. (Cited in Nitta, 1997).

[14] Li, Y., Delsante, A., (2001) Natural Ventilation Induced by Combined Wind and Thermal Forces, Building and Environment 36: 59-71, http://dx.doi.org/10.1016/S0360-1323(99)00070-0

[15] Li, Y., Delsante, A., Chen, Z., Sandberg, M., Andersen, A., Bjerre, M. and Heiselberg, P., (2001) Some Examples of Solution Multiplicity in Natural Ventilation, Building and Environment 36: 851-858, http://dx.doi.org/10.1016/S0360-1323(01)00011-7

[16] Hunt, G.R. Linden, P.F. (2004) Displacement and Mixing Ventilation Driven by Opposing Wind and Buoyancy, Journal of Fluid Mechanics 527: 27-55, http://dx.doi.org/10.1017/S0022112004002575 
[17] Marchant, E.W., (1984) Effect of Wind on Smoke Movement and Smoke Control Systems, Fire Safety Journal 7:55-63, http://dx.doi.org/10.1016/0379-7112(84)90008-0

[18] Heskestad, G. "Fire Plume Air Entrainment according to two Competing Assumptions," 21th Symposium on Combustion, The Combustion Institute, Pittsburgh, 1986.

[19] Strogatz, S.H., Nonlinear Dynamics and Chaos - with Applications to Physics, Biology, Chemistry, and Engineering, Addison-Wesley, Reading, 1994.

[20] Hunt, G.R. and Linden, P.F., (2001) Steady-state Flows in an Enclosure Ventilated by Buoyancy Forces Assisted by Wind, Journal of fluid mechanics 426: 355-386, http://dx.doi.org/10.1017/S0022112000002470

[21] Perko, L., Differential Equations and Dynamical Systems, New York: Springer-Verlag, 1993. 